\title{
A WIRELESS LOW-RANGE PRESSURE SENSOR BASED ON P(VDF-TRFE) PIEZOELECTRIC RESONANCE
}

\author{
A Thesis \\ Presented to the Faculty of the Graduate School \\ of Cornell University \\ In Partial Fulfillment of the Requirements for the Degree of \\ Master of Science
}

by

Xiaoyang Li

January 2011 
(C) 2010 Xiaoyang Li 


\begin{abstract}
A low-range bio-compatible pressure sensor based on $\mathrm{P}(\mathrm{VDF}-\mathrm{TrFE})$ piezoelectric resonance has been tested and integrated to a wireless platform. Many critical physiological applications and in-vivo diagnosis require highly sensitive pressure monitoring in the $0-100 \mathrm{kPa}$ range, with negligible long-term drift and robust integration platform. The PVDF film and its copolymers exhibit significant piezoelectric properties after poling. $\mathrm{P}(\mathrm{VDF}-\mathrm{TrFE})$ is preferred for its flexible film structure, low modulus, high yield strength and resistance to corrosive chemicals. All piezoelectric materials are lossy and thus the leakage current will cause drift of instantaneous response, which limits the direct use of piezoelectric charges in sensing. In this paper, the pressure sensor which adopts the appropriate properties of P(VDFTrFE) for low pressure levels, works as an acoustic wave resonator to avoid long-term drift from the charge leakage problem in quasi-static pressure, while maintaining good sensitivity. Simulation results show good agreement with the measurement data. The temperature coefficient and the areal scaling effect are characterized. Furthermore, the sensor output is matched with standard $50 \Omega$ impedance at the resonance for integration into the RF circuits. A passive sensor tag is demonstrated by using RF backscattering.
\end{abstract}




\section{BIOGRAPHICAL SKETCH}

Xiaoyang Li was born in Luohe, People’s Republic of China, in October 1985. He attended elementary school and middle school in Luohe. Then he went to Tsinghua University, Beijing, China since August 2003. During his undergraduate studies, he attended the Department of Electronic Engineering. From August 2005 to January 2006, he enrolled the undergraduate exchange program with Hong Kong University of Science \& Technology, Hong Kong. In July 2007 he graduated from Tsinghua University with Bachelor of Engineering degree. Later on in August 2007, he entered the MS/PhD program at School of Electrical \& Computer Engineering, Cornell University for graduate studies. He worked with Professor Kan in the research area of pressure sensor and sensor integration related projects. 
To Mei Zhong 


\section{ACKNOWLEDGMENTS}

I would like to thank Professor Kan for giving me the valuable chance to work in his research group and providing the financial support for my graduate studies. I thank Professor Apsel and Professor Bhave for being my special committee members and reviewing my thesis. I also thank Professor Afshari for kindly being the proxy of the exam.

I would like to thank my colleagues Fan Yu, Rajeev Dokania, Guansheng Li, Timothy

Reissman, Bo Xiang, Xuan Silvia Zhang, Xiao Wang, Arun Swain and all the Professor Kan’s group members for insightful advices and friendship. 
TABLE OF CONTENTS

BIOGRAPHICAL SKETCH iii

DEDICATION iv

ACKNOWLEDGMENT $\mathrm{V}$

TABLE OF CONTENTS vi

LIST OF FIGURES vii

LIST OF TABLES ix

CHAPTER 1: INTRODUCTION 1

CHAPTER 2: P(VDF-TRFE) PIEZOELECTRIC PRESSURE SENSOR MODELING AND MEASUREMENT

2.1 Theory 5

2.2 Impedance measurement 10

2.3 The scaling effect 12

2.4 The temperature drift 13

2.5 Wireless transmission 14

2.6 Long-term stability 20

CHAPTER 3: CONCLUSION AND FUTURE WORK 23

REFERENCES 25 


\section{LIST OF FIGURES}

Figure 1: SEM of $\mathrm{P}(\mathrm{VDF}-\mathrm{TrFE})$ piezoelectric film in a cross-section view..........3 Figure 2: The modified BVD equivalent circuit model including parasitics for the piezoelectric resonator. $L_{1}$ and $R_{1}$ account for the fluid loading effect on the pressure

sensor.................................................................6

Figure 3: Sensor impedance magnitude and phase comparison between measurement and simulation. The measurement is done under atmosphere pressure..............9

Figure 4: Pressure sensor experiment setup.................................10

Figure 5: The measured sensor impedance magnitude and phase under various pressure levels.....................................................11

Figure 6: The relation between $L_{2}$ and air pressure. By equation in Table $1, L_{2}$ is

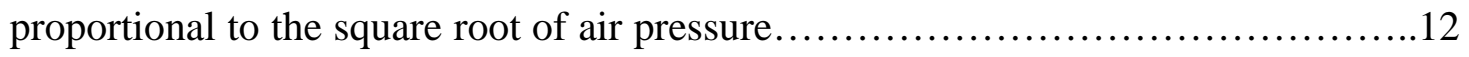
Figure 7: Geometrical scaling effect of $\mathrm{P}(\mathrm{VDF}-\mathrm{TrFE})$ film lateral dimensions on pressure sensor sensitivity (16x refers to area). The measurement data shows that the sensitivity has little variations.............................................

Figure 8: The temperature drift characteristic of sensor resonance frequency.........14 Figure 9: Measured $S_{11}$ magnitude and phase of the pressure sensor after impedance compensation........................................................ 15

Figure 10: Wireless transmission scheme for the passive pressure sensor tag........17 Figure 11: Demonstration of sensor wireless transmission by the received PSD at the

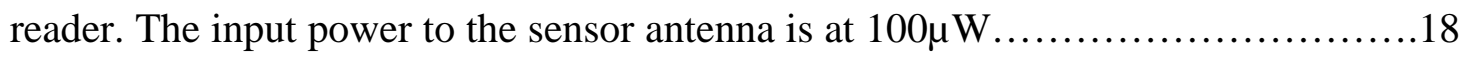

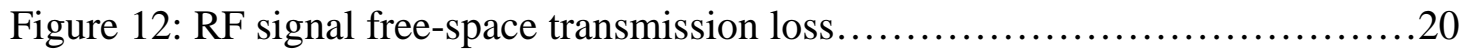

Figure 13: Pressure sensor structure with the P(VDF-TrFE) film.................20 Figure 14: The schematic for measurement. The oscilloscope provides the

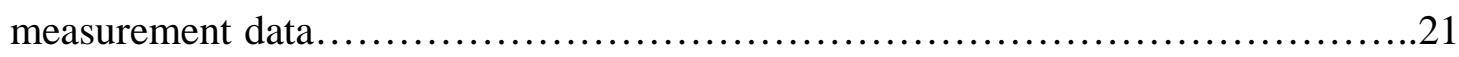


Figure 15: The long-term stability monitored by the RMS voltage reading near

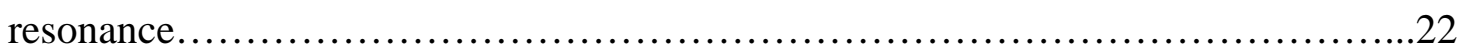




\section{LIST OF TABLES}

Table 1 BVD model parameter equations.....................................7

Table 2 Typical material constant values and fitting parameters for the BVD model....................................................................... 8 


\section{CHAPTER 1}

\section{INTRODUCTION}

With the development of modern techniques, low-range pressure sensing now has many critical applications in biomedical devices and human-machine interface equipment. The market keeps increasing rapidly and the demand for high sensitivity in the low pressure range, negligible long-term drift and robust integration platform requires a lot of dedicated efforts in this area. Many research groups have explored different design methods and a large number of results on the low-range pressure sensor have been published.

The MEMS-based capacitive pressure sensor has already been demonstrated in many applications. It usually consists of a variable diaphragm-substrate capacitor, two electrodes on both top and bottom surfaces of the capacitor, and a reference pressure cavity. The diaphragm deflects when exposed at different pressure between reference and the volume under investigation, which causes capacitance change. The authors explained the fabrication process of capacitive pressure sensor using silicon integrated circuit technology in [1]. Further improvement on sensor fabrication and performance are discussed in [2-4]. In [5], the pressure sensor is integrated into a specifically designed micro-system, which includes both data acquisition and data processing modules. However, in-vivo pressure sensing requires mechanical robustness and the capacitive pressure sensor is usually kind of fragile due to the thin membrane structure and requires complex. The requirement for reference pressure also brings difficulty for the sensor assembly and packaging. The rubber-based capacitive pressure sensor improves on resistance to environmental shock or vibration, but has the rubber material aging concern [6], which is not suitable for human implantation. The piezoresistive materials exhibit resistance variation due to applied stress. However, the 
piezoresistive pressure sensor is limited for its low sensitivity [7] in this pressure range.

For sensors based on piezoelectric materials, the application of stress on the material results in dipole charge formation and electrical potential difference on the surfaces, which is called piezoelectric effect. A variety of tactile sensors have been made based on this effect [8-10]. However, these sensors can only monitor instantaneous stress change. In consideration of the long-term operation, all of the piezoelectric materials are electrically lossy. If the applied stress is static or at very low-frequency, the piezoelectric charges will decay gradually because of the non-zero conductivity of the materials [11-13], and the important transducing information cannot be recovered. To avoid the long-term drift by the leakage current, the piezoelectric resonators in the thickness mode are often chosen as the sensing element. Piezoelectric materials stimulated by external AC power resonate at a particular resonance frequency, which is determined by the material physical properties. The resonance frequency and the quality factor will decrease under the mass or fluid loading effect [14]. The resonance frequency shift is the indication of the applied stress change. However, this is difficult for low-range pressure sensing if the modulus of the used material is high.

Polyvinylidene fluoride (PVDF) and its copolymers have very low modulus among all the piezoelectric materials. Some published works have discussed the possibility of using PVDF as pressure sensor. In [15], the PVDF film has been used in a static force sensor, and a mechanical model has been derived. Poly(vinylidene fluoride - trifluoroethylene) ( $\mathrm{P}(\mathrm{VDF}-\mathrm{TrFE})$ ) is one of its most commonly-used copolymers, which is preferred for its high charge constant, flexible film structure, low modulus, high yield strength and resistance to corrosive chemicals. 
In our experiment setup, the poled $50 \mu m \pm 1 \mu \mathrm{m}$ thickness $\mathrm{P}(\mathrm{VDF}-\mathrm{TrFE})$ copolymer film as the transducing material has been sputtered gold on both top and bottom layers for electrodes. The scanning electron microscopy (SEM) of the film cross-section is shown in Figure 1.

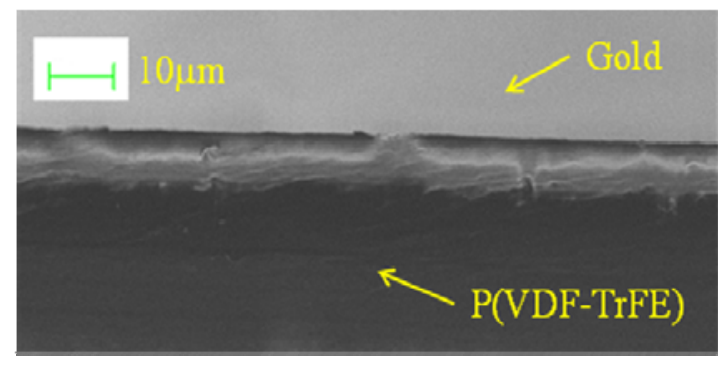

Figure 1: SEM of P(VDF-TrFE) piezoelectric film in a cross-section view.

The mobile biomedical diagnostic device often needs wireless transmission. In [16], the standard on-chip analog-digital converter (ADC), voltage controlled oscillator (VCO) and digital modulators/demodulators were employed in the data link. The sensor analog outputs are first converted to digital forms by ADC, which are sent out through RF transmitter. These complex circuits consume significant chip areas and the ADCs are usually very power-hungry. Designs that use a battery are difficult to miniaturize to maintain the long-term operation. An alternative approach is to use a RF-DC converter to collect power from electromagnetic fields and a large off-chip capacitor to store energy. However, the sensor system size will still be significant. To overcome these problems, we employ the RF-backscatter scheme as a passive sensor tag. The pressure sensor is modified to match with standard $50 \Omega$ impedance at the resonance for integration.

In this thesis, we explored the pressure sensor properties in fluidic environment and used an equivalent circuit model for guiding the design process in Chapter 2. The circuit model matched well with the measurements. In Chapter 2, a variety of pressure sensor measurements have been performed. The areal scaling was performed and was 
found to have minimal effects on sensitivity. The temperature coefficient of resonance frequency was also characterized. The pressure sensor wireless transmission is also discussed. The conclusion and future work is briefly discussed in Chapter 3. 


\section{CHAPTER 2}

\section{P(VDF-TRFE) PIEZOELECTRIC PRESSURE SENSOR MODELING AND MEASUREMENT}

\subsection{Theory}

When a mechanical stress is applied, the piezoelectric material produces an electric dipole. Conversely, a mechanical deformation is produced when an electric field is applied to the material. Under exposure to an AC electric field, the piezoelectric element changes its dimensions periodically at the cycling frequency of the field. At the resonance frequency the piezoelectric element converts the input electrical energy into mechanical energy most efficiently.

The piezoelectric behavior can be characterized by the equivalent circuit of the Butterworth-Van Dyke (BVD) model [17-26] in Figure 2. $C_{1}, L_{l}$ and $R_{l}$ known as the "the mechanical arm", describe the mechanical vibrations resulting from the piezoelectric effect with a finite quality factor. $C_{0}$ represents the bulk capacitance due to the electrodes affixed to the $\mathrm{P}(\mathrm{VDF}-\mathrm{TrFE})$ film, which is also called 'shunt' capacitance. $R_{0}$ accounts for the bulk resistance across the film. $L_{p}$ and $R_{p}$ are the

parasitic from the packaging pads. In our setup, $L_{p}$ dominates the parasitic while $R_{p}$ is almost negligible. 


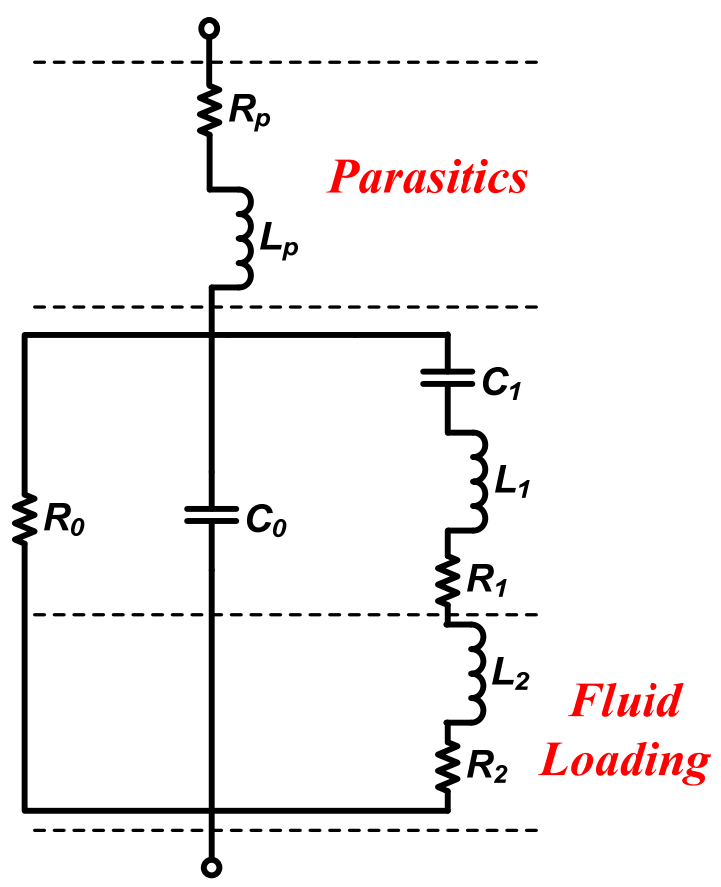

Figure 2: The modified BVD equivalent circuit model including parasitics for the piezoelectric resonator. $L_{1}$ and $R_{1}$ account for the fluid loading effect on the sensor.

As the frequency of the electrical field increases, the resonator impedance first approaches a minimum value (maximum admittance). This frequency is called the series resonance frequency $\omega_{s}$, which is controlled by the motional branch $C_{1}, L_{1}$ and $R_{l}$ and is determined by the properties of the piezoelectric material and the physical dimensions. Generally for the thickness mode, a thicker element has a lower resonance frequency. As the frequency is further increased, impedance increases to a maximum (minimum admittance). The maximum impedance frequency is named as the parallel resonance frequency $\omega_{p}$. Both $\omega_{s}$ and $\omega_{p}$ are located at the frequencies where the first derivatives of the impedance magnitude equal to zero.

When the film is in contact with fluid, it will generate acoustic waves into the fluid and cause the acoustic energy to radiate away. The viscosity and pressure coupling of the surrounding medium results in the decrease of both the resonance 
frequency $\omega_{s}$ and quality factor $Q . L_{2}$ and $R_{2}$ are included in the BVD model to account for this loading effect.

When all the parameters and geometry sizes are known, the resonator can be characterized by the physical equations in Table 1 where $\varepsilon_{r}$ is the relative dielectric constant, $A$ is the film area, $K_{0}$ is the electromechanical coupling factor, $n$ is the harmonic order, $h$ is the film thickness, $E_{p}$ is the effective elastic modulus, $\rho_{q}$ is the film density, $\eta$ is the viscosity of contacting fluid, $\rho$ is the fluid density, and $\eta_{q}$ is the effective film viscosity.

Table 1: BVD model parameter equations.

\begin{tabular}{|c|c|c|}
\hline$C_{0}$ & $L_{1}$ & $C_{1}$ \\
\hline$C_{0}=\frac{\varepsilon_{r} \varepsilon_{0} A}{h}$ & $L_{1}=\frac{1}{\omega_{s}{ }^{2} C_{1}}$ & $C_{1}=\frac{8 K_{0}{ }^{2} C_{0}}{(n \cdot \pi)^{2}}$ \\
\hline$R_{1}$ & $L_{2}$ & $R_{2}$ \\
\hline$R_{1}=\frac{\eta_{q}}{E_{p} C_{1}}\left(\frac{\omega}{\omega_{s}}\right)^{2}$ & $L_{2}=\frac{\omega_{s} L_{1}}{n \cdot \pi}\left(\frac{2 \rho \eta}{\omega E_{p} \rho_{q}}\right)^{1 / 2}$ & $R_{2}=\frac{\omega_{s} L_{1}}{n \cdot \pi}\left(\frac{2 \omega \rho \eta}{E_{p} \rho_{q}}\right)^{1 / 2}$ \\
\hline
\end{tabular}

Initial values for $L_{1}$ and $C_{1}$ can be calculated by equations in Table 1 and material constants in Table 2. The exact fitting values are obtained by curve fitting using simulated annealing. The fitting parameter values are also listed in Table 2 [15]. 
Table 2: Typical material constant values and fitting parameters for the BVD model.

\begin{tabular}{|c|c|c|c|c|}
\hline$\omega_{s}$ & $\rho_{q}$ & $K_{0}$ & $\varepsilon_{r}$ & $E_{p}$ \\
\hline $\begin{array}{c}1.388 \times 10^{8} \\
\mathrm{rad} / \mathrm{s}\end{array}$ & $\begin{array}{c}1.86 \times 10^{3} \\
\mathrm{~kg} / \mathrm{m}^{3}\end{array}$ & $11 \%$ & 12 & $9.1 \mathrm{GPa}$ \\
\hline$h$ & $C_{l}$ & $L_{1}$ & $R_{l}$ & $n$ \\
\hline $50 \mu \mathrm{m}$ & $1.34 \mathrm{pF}$ & $38.43 \mu \mathrm{H}$ & $415 \Omega$ & 1 \\
\hline
\end{tabular}

The most sensitive frequency point $\omega_{i}$ lies between $\omega_{s}$ and $\omega_{p}$. Equation 1 describes the resonator impedance without the parasitic. As $R_{0}$ is very large and invariable to frequency, it is reasonable to ignore it here. Equations 2 and 3 show the $\omega_{s}$ and $\omega_{p}$ expressions when neglecting the influence of $R_{l}$ on resonance frequency. $\omega_{i}$ is approximately the mean of $\omega_{1}$ and $\omega_{2}$. The analytical solution of $\omega_{i}$ in Equation 4 can help design better signal processing blocks for the sensor.

$$
\begin{gathered}
Z_{\text {in }}=\frac{L_{1} C_{1} s^{2}+R_{1} C_{1} s+1}{\left(C_{1}+C_{0}\right) \cdot s \cdot\left(\frac{L_{1} C_{1}}{\left.1+\frac{C_{1}}{C_{0}} s^{2}+\frac{R_{1} C_{1}}{1+\frac{C_{1}}{C_{0}}} s+1\right)}\right)} \\
\left.\omega_{s}=\sqrt{\frac{1}{L_{1} C_{1}}}\right) \\
\omega_{p}=\sqrt{\frac{1}{L_{1} C_{1}} \cdot\left(1+\frac{C_{1}}{C_{0}}\right)}\left(\frac{\pi}{2 h} \sqrt{\frac{E_{p}}{\rho_{q}}} \cdot\left(1+\sqrt{1+\frac{8 K_{0}^{2}}{\pi^{2}}}\right) \cong \frac{\pi}{h} \sqrt{\frac{E_{p}}{\rho_{q}}} \cdot\left(1+\frac{2 K_{0}^{2}}{\pi^{2}}\right)\right.
\end{gathered}
$$

To verify the BVD model, a $1 \mathrm{~cm}$ by $1.5 \mathrm{~cm}$ area $\mathrm{P}(\mathrm{VDF}-\mathrm{TrFE})$ film is taken as the sample for experiment. The lead wires are connected to pressure sensor with conductive epoxy. The impedance measurement in the atmosphere pressure $(101 \mathrm{kPa}$ at room temperature) is done by an impedance analyzer HP4194A and compared with the simulation results from the BVD model. Good agreement has been achieved as shown in Figure 3. Due to the large resistive loss by $R_{l}$, the P(VDF-TrFE) film 
resonator is a low- $Q$ resonator [27], which is manifestly below 15 in the measurement. As a tradeoff, materials which have higher quality factor such as Aluminum Nitride or PZT [28] have larger elastic modulus, which are not sensitive enough in the pressure range we are interested in.
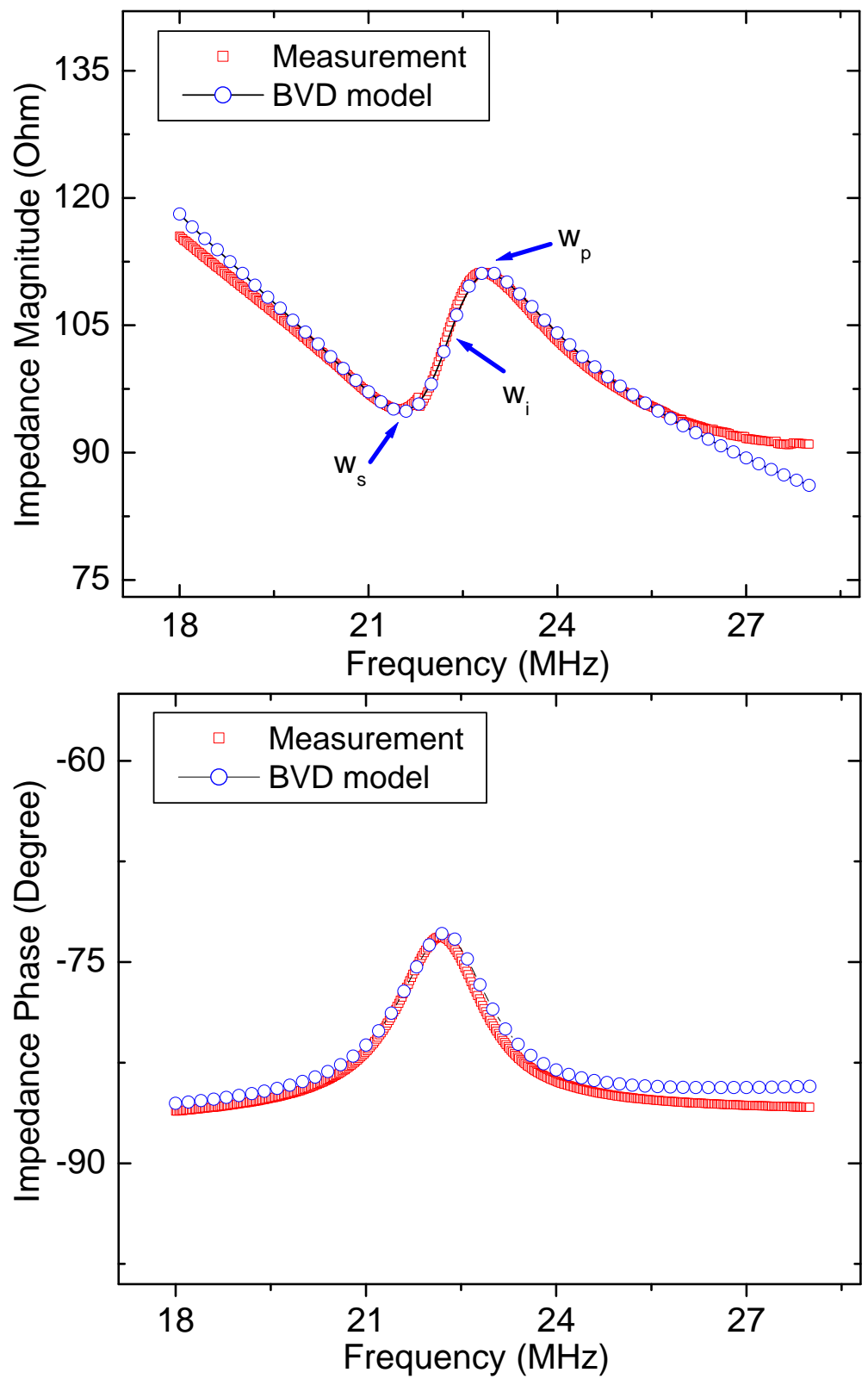

Figure 3: Sensor impedance magnitude and phase comparison between measurement and simulation. The measurement is done under atmosphere pressure. 


\subsection{Impedance measurement}

The pressure test was performed in a vacuum chamber. Figure 4 illustrates the setup. The pressure sensor is mounted inside the chamber and the lead wires go through the chamber lid via holes sealed with silicone. A vacuum pump connected with the chamber controls the air pressure level of inside environment. The sensor impedance characteristic was monitored by the impedance analyzer.

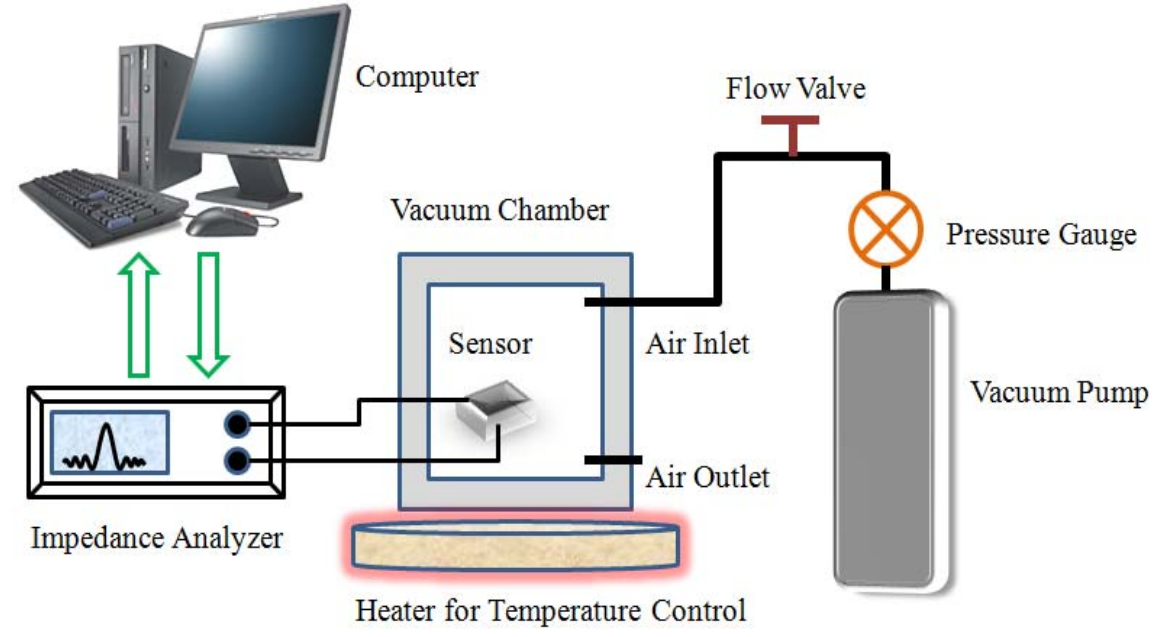

Figure 4: Pressure sensor experiment setup.

Figure 5 shows how the impedance real and imaginary parts change under different pressure. The measurement accuracy (error rate) is $\pm 5 \mathrm{kPa}$ per point. This accuracy is presently limited by the impedance analyzer resolution. 

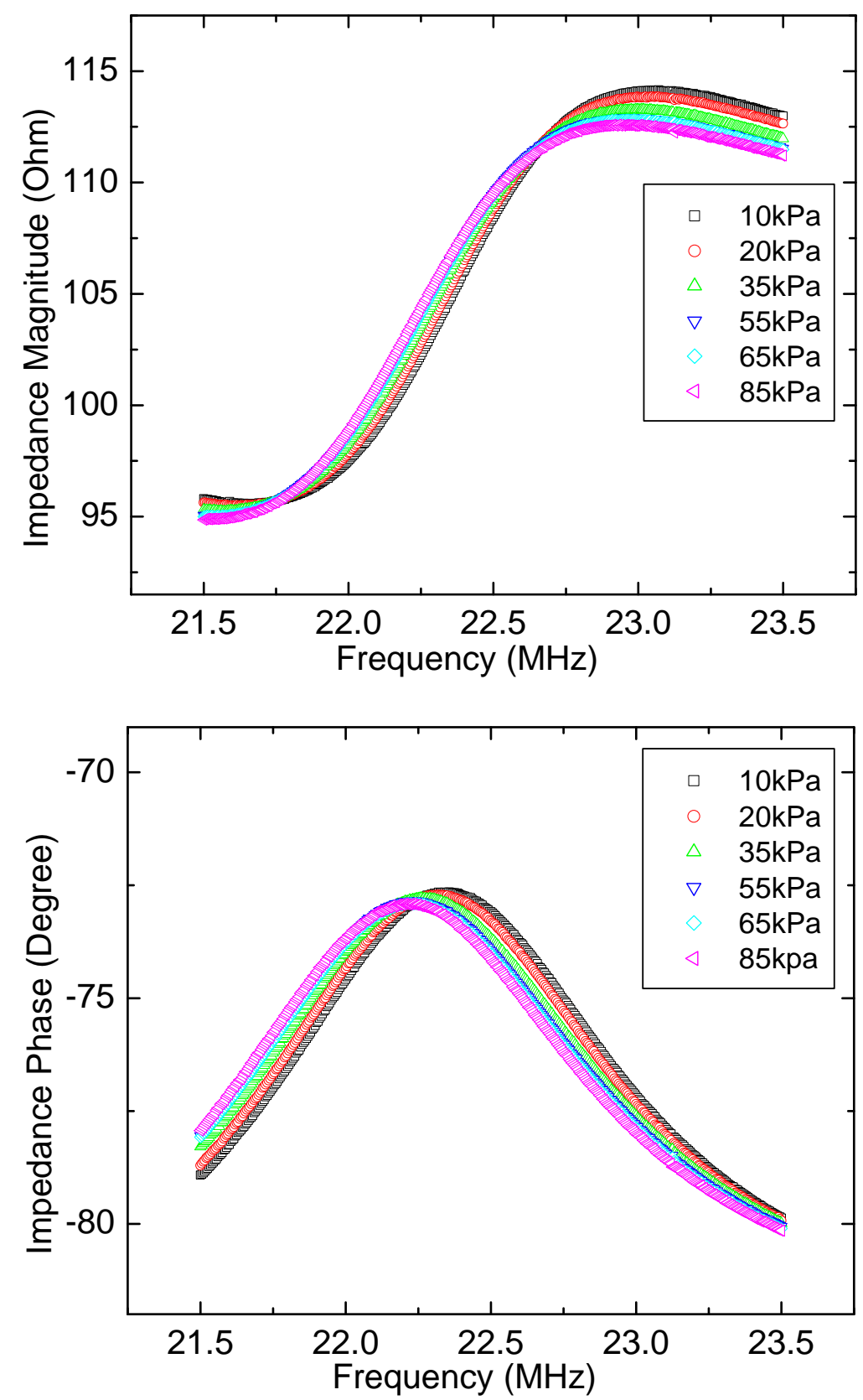

Figure 5: The measured sensor impedance magnitude and phase under various pressure levels.

Based on the BVD model, the extracted values of $L_{2}$ from measurement are close to the calculated results using equations in Table 1, as shown in Figure 6. We 
can observe that $L_{2}$ is proportional to the square root of the compressible fluid density, which is directly related to the pressure.

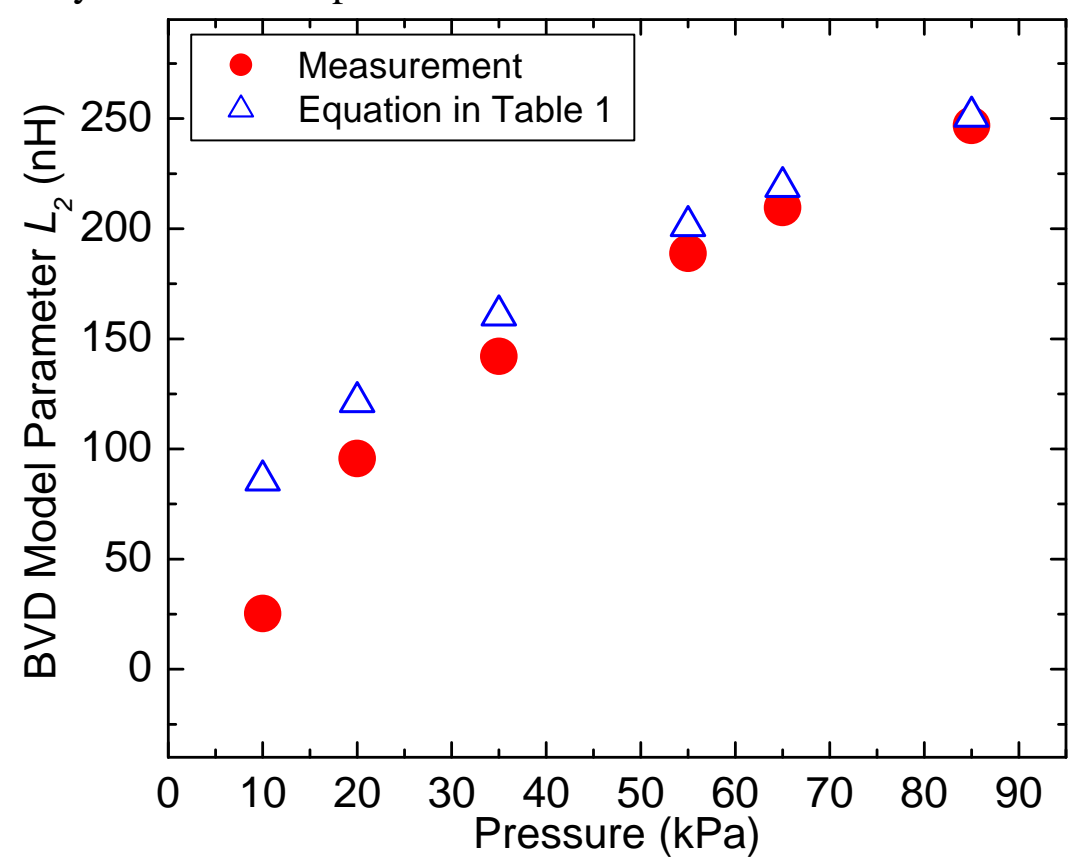

Figure 6: The relation between $L_{2}$ and air pressure. By equation in Table $1, L_{2}$ is proportional to the square root of air pressure.

\subsection{The scaling effect}

The scaling effect of the sensor has also been studied in our experiment. The film thickness is kept at $50 \mu \mathrm{m}$ and the lateral dimension is decreased gradually. The geometry sizes of the sensor film that are tested range from $1 \mathrm{~cm}$ by $1.5 \mathrm{~cm}$ down to $25 \mathrm{~mm}$ by $38 \mathrm{~mm}$. The resonance frequency shift under pressure is recorded with 5 different sizes to track the sensitivity. The test results in Figure 7 show that the variation of lateral size has negligible effects on the pressure sensitivity. As the resonance frequency is mainly determined by the thickness of the film for the thickness mode, unless the length or width of the film is comparable to the thickness. The scaling test illustrated that direct integration on silicon chip is feasible. From 
Figure 7, we can also observe that the sensitivity gradually saturates as the pressure increases, which has been reported by [17].

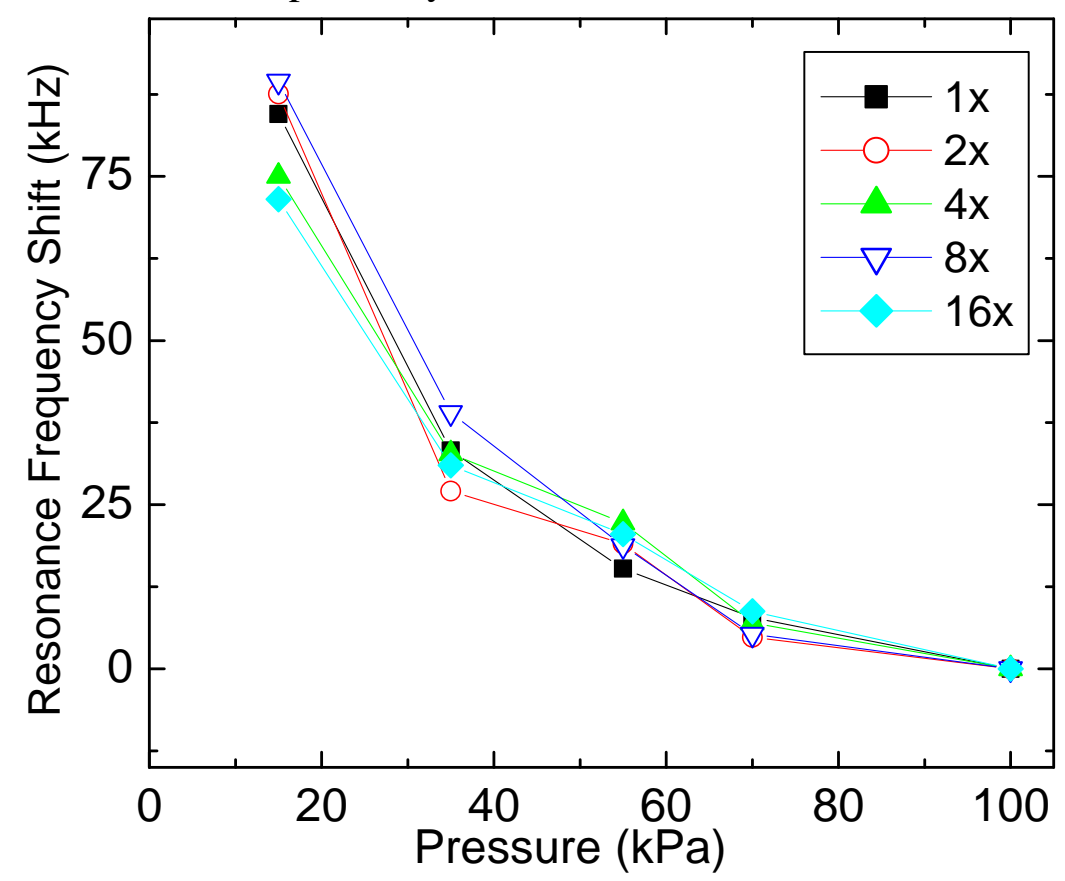

Figure 7: Geometrical scaling effect of $\mathrm{P}(\mathrm{VDF}-\mathrm{TrFE})$ film lateral dimensions on pressure sensor sensitivity (16x refers to area). The measurement data shows that the sensitivity has little variations.

\subsection{The temperature drift}

The temperature drift characteristic of pressure sensor is a very important parameter. For human body pressure measurement, the temperature range is relatively stable around $37 \pm 0.5^{\circ} \mathrm{C}$. The variation is almost negligible when people are in healthy status (as well as for mammals in veterinary monitoring). We extend the temperature measurement range to calculate the temperature shift coefficient. The temperature measurement data will also be useful for compensation in vitro sensing. The vacuum chamber is heated by a heat gun to control the temperature varying from $23^{0} \mathrm{C}$ to $46^{0} \mathrm{C}$. The measured temperature drift characteristic is shown in Figure 8, where the 
resonance frequency decreases as the temperature increases. The curve fitting reveals that the resonance frequency is almost linearly dependent on the temperature. The fluid viscosity $\eta$ has a strong dependence on temperature, which will affect the $L_{2}$ term besides the air pressure.

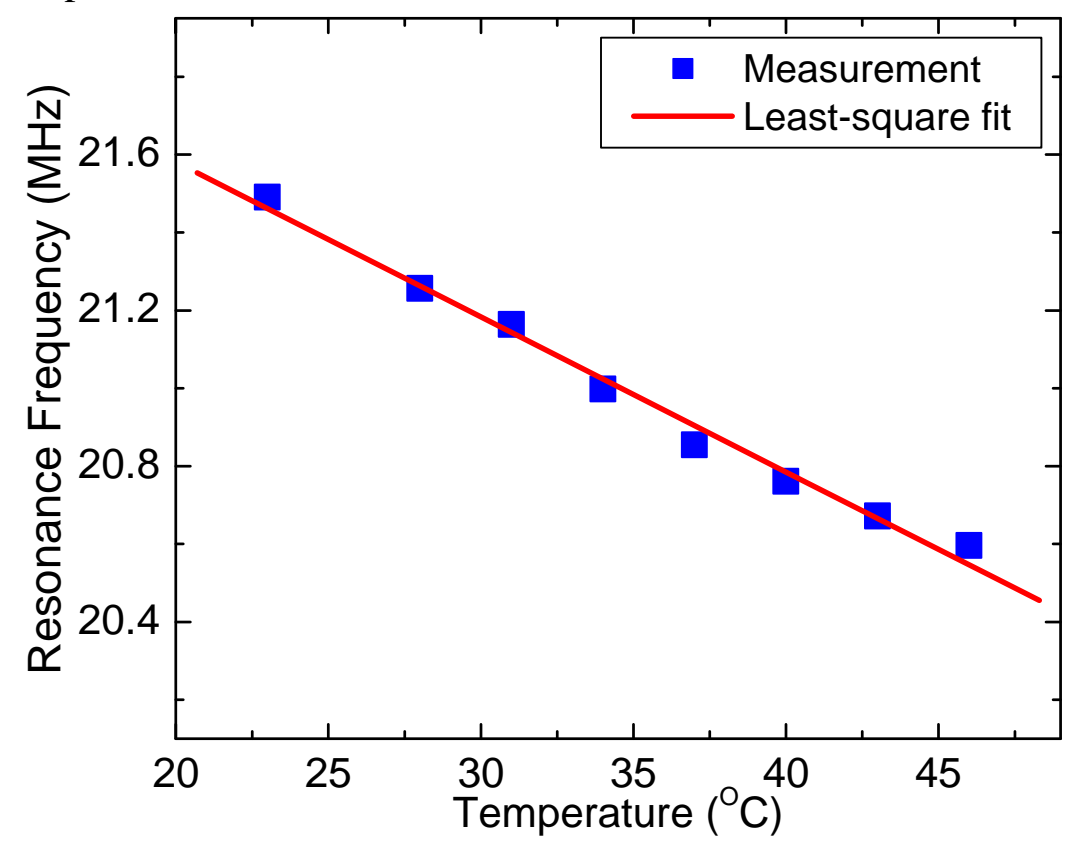

Figure 8: The temperature drift characteristic of sensor resonance frequency.

In applications with large temperature variations, compensation to the temperature shift will require an independent temperature sensor to calibrate the readout at the post-processing stage. Differential measurement could also help to eliminate the temperature effect. Two identical sensors can be assembled, and pressure is applied on one of them and the other one free standing. Both of them are exposed in the environment with the same temperature. The differential readout will cancel out the temperature drift.

\subsection{Wireless transmission}

Starting with the simulation results and extracted parameters from the BVD model, the sensor is impedance matched with the standard $50 \mathrm{ohm}$ resistance at the resonance on the customized PCB to integrate with the RF circuits. The customized PCB is a single 
layer printed circuit board. The P(VDF-TrFE) film is attached on the top surface of PCB by epoxy. Multiple SMT soldering pads in series / parallel connection with the film are provided on the PCB. A 26 ohm resistor and a $10 \mathrm{nH}$ inductor in series connection with the $\mathrm{P}(\mathrm{VDF}-\mathrm{TrFE})$ film are used for impedance match with $50 \mathrm{ohm}$ at the resonance frequency. A SMA connector is built on the board edge to connect with the network analyzer. The PCB here is designed only for experimental verification purpose, not for eventual application packaging, where a custom design would be necessary.

As shown in Figure 5, the ambient pressure causes impedance change and resonance frequency shift. Thus the $S_{11}$ parameters at the input port of the sensor will reflect the pressure as well. Figures 9 shows the measured $S_{11}$ phase and magnitude shift with pressure. The measurement is done by the network analyzer Agilent E8364B.

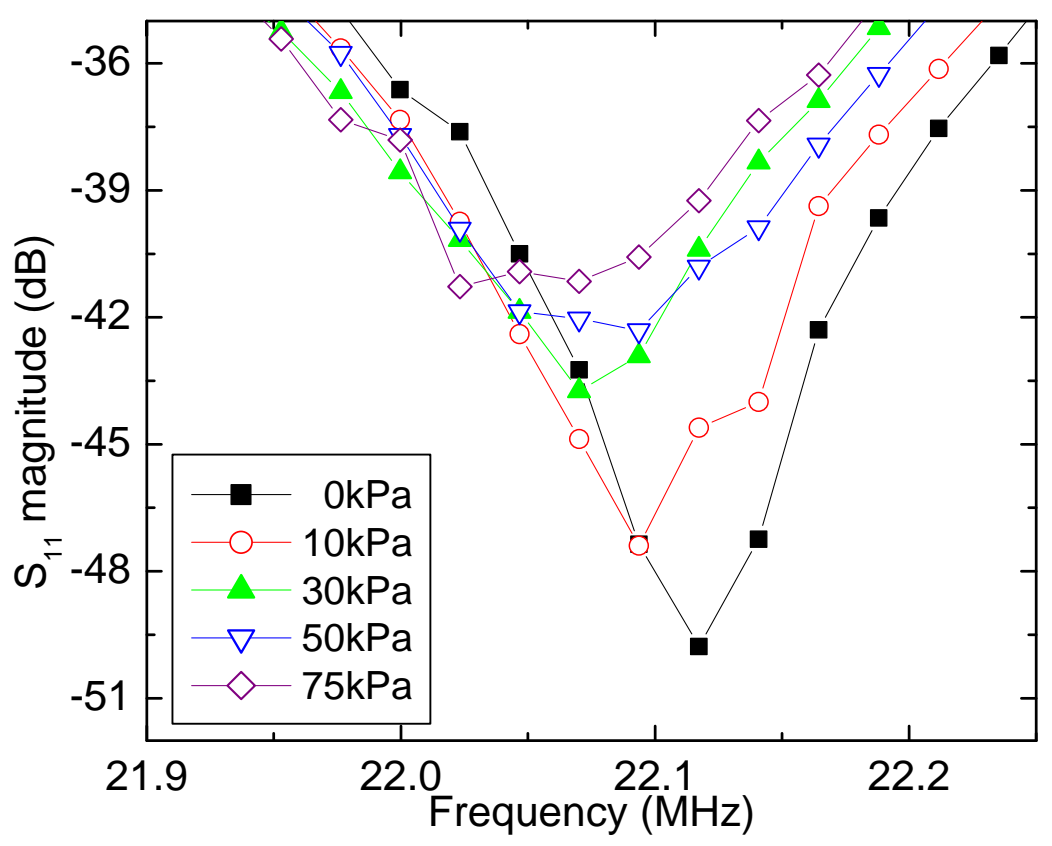

Figure 9: Measured $S_{11}$ magnitude and phase of the pressure sensor after impedance compensation. 
Figure 9 (Continued)

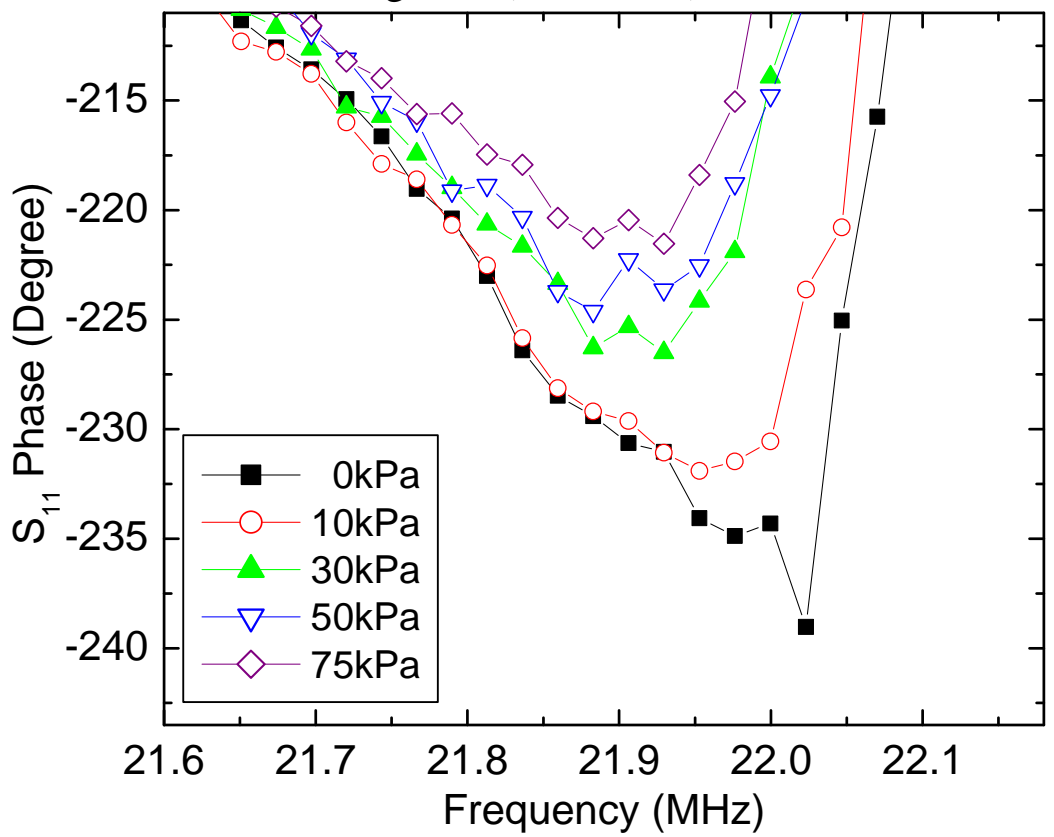

In [29-31], it was demonstrated that the reflected signal frequency or time domain information changes with $S_{11}$. By modulating the $S_{11}$ magnitude or phase, pressure information can be effectively transmitted through the RF signal. From the measurements in Figure 9, it is possible to use a single test frequency for the wireless sensing, where the most sensitivity by $S_{11}$ loss or phase shift can be achieved at the unloaded resonant frequency around 22MHz. The amplitude coding is more vulnerable to the ambient reflection. A broadband signal transmission can then be preferred. The reader in the present setup uses only one antenna for the data transmission operation. First, a broadband pulse wave is generated and transmitted by the reader antenna to the antenna connected with the pressure sensor tag. The pulse signal should have a flat spectrum profile at least in the frequency range of $22 \pm 0.5 \mathrm{MHz}$. A Gaussian function or Sinc function pulse with less than 40ns duration would satisfy this condition. Part of the transmitted power could be received by the sensor tag antenna. Due to the impedance mismatch between sensor and sensor tag 
antenna, the backscattered power is re-radiated by the sensor tag antenna. Then the backscattered pulse signal from the sensor tag, which is determined by the $S_{11}$, will be captured by the reader antenna. By comparing the Power Spectrum Density (PSD) transmitted and received by the reader antenna, we can readily retrieve the sensor impedance information. This is a classical RFID backscattering setup. The wireless transmission scheme is illustrated in Figure 10.

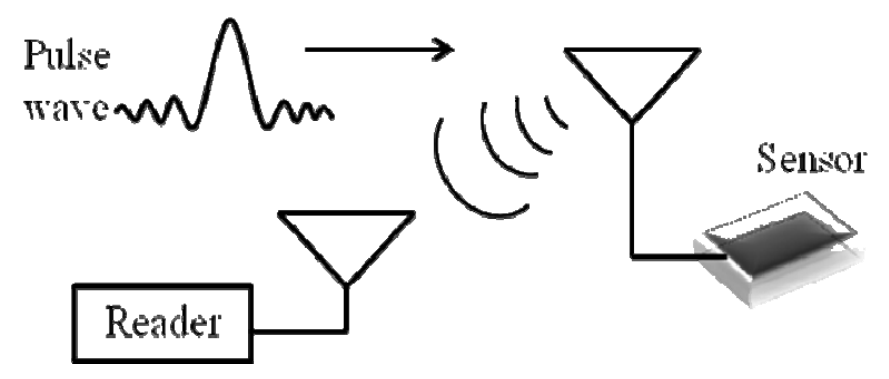

Figure 10: Wireless transmission scheme for the passive pressure sensor tag.

RF system-level simulation is done to verify this protocol. The signal transmission is simulated in Cadence with the SPICE models [32-33]. The signal PSD at the reader antenna is shown in Figure 11. The simulation results demonstrate the possible application of our pressure sensor in the passive telemetry system. 


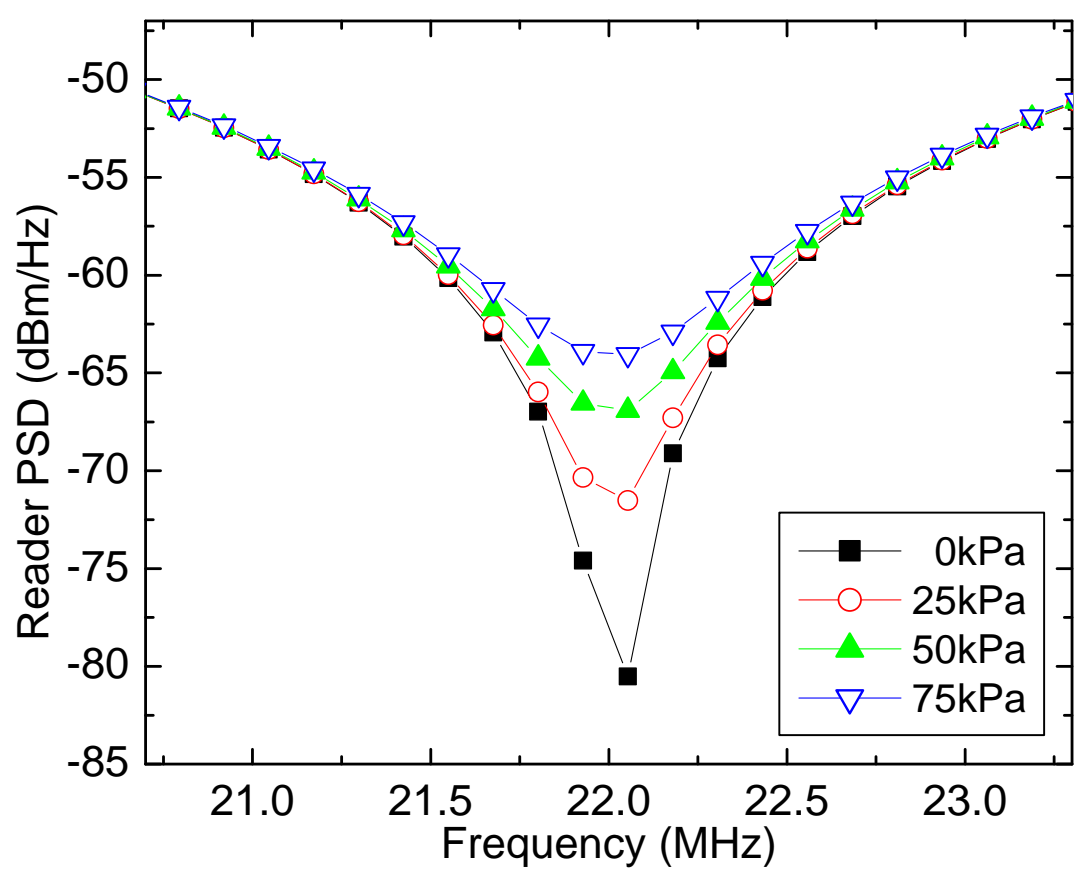

Figure 11: Demonstration of sensor wireless transmission by the received PSD at the reader. The input power to the sensor antenna is at $100 \mu \mathrm{W}$.

The power $P_{a}$ collected by the sensor tag antenna is defined by the product of the power density of the electromagnetic wave incident to the sensor tag antenna in the free space and the antenna effective area [34]:

$$
P_{a}=P_{\text {transmitted }} \frac{G_{r}}{4 \pi d^{2}} A_{e}
$$

Where $P_{\text {transmitted }}$ is the transmitted power by reader antenna, $G_{r}$ is the gain of the reader antenna, and $d$ is the distance from reader to the sensor tag, $A_{e}$ is the effective area of the antenna given by

$$
A_{e}=\frac{\lambda^{2}}{4 \pi} G_{s}
$$

Where $\lambda$ is the electromagnetic wavelength, $G_{s}$ is the gain of the sensor tag antenna. The power re-radiated by the sensor tag in the direction of reader can be found by

$$
P_{\text {re-radiated }}=\delta_{\text {load }} P_{a} G_{s}
$$

Where the factor $\delta_{\text {load }}$ is given by

$$
\delta_{\text {load }}=\frac{4 R_{a}^{2}}{\left|R_{a}+Z_{\text {load }}\right|^{2}}
$$


Where $R_{a}$ is the antenna characteristic impedance, $Z_{l o a d}$ is the sensor load impedance. $\delta_{\text {load }}$ defines the ratio of sensor tag backscattered power to the collected power, which reflects the influence of the tag load impedance on the re-radiated power.

$$
P_{\text {received }}=P_{\text {transmitted }} \frac{G_{r}{ }^{2} G_{s}{ }^{2} \lambda^{4} \delta_{\text {load }}}{(4 \pi)^{4} d^{4}}=P_{\text {transmitted }} \frac{G_{r}{ }^{2} G_{s}{ }^{2} C^{4} \delta_{\text {load }}}{(4 \pi)^{4} d^{4} f^{4}}
$$

Where $C$ is the traveling speed of electromagnetic wave, $P_{\text {received }}$ is the power received by the reader antenna from the sensor tag antenna backscattered energy.

The power loss can be characterized by the ratio

$$
20 \log \frac{P_{\text {received }}}{P_{\text {transmitted }}}=20 \log \frac{G_{r}{ }^{2} G_{s}{ }^{2} C^{4} \delta_{\text {load }}}{(4 \pi)^{4} r^{4} f^{4}}=20 \log \frac{G_{r}{ }^{2} G_{s}{ }^{2} C^{4} \delta_{\text {load }}}{(4 \pi)^{4} r^{4}}-80 \log f
$$

The equation above describes how the free space loss, distance variation and frequency would affect the backscattered power received by the reader antenna. From reference [35], the antenna bandwidth can be more than $50 \%$ or even $100 \%$ of the center frequency, which is more than sufficient for us. And during the data transmission, the location of reader and sensor tag are fixed. We assume $\delta_{\text {load }}$ is relatively constant when we are considering the transmission loss. The loss mainly depends on the frequency change.

The sensor resonance frequency is around $22 \mathrm{MHz}$, and the change range is typically within $0.1 \mathrm{MHz}$. Figure 12 illustrates how the loss changes within the frequency range from $21 \mathrm{MHz}$ to $23 \mathrm{MHz}$ ( $20 \mathrm{MHz}$ is selected as $0 \mathrm{~dB}$ reference point). The transmission loss is negligible within the $22 \pm 0.1 \mathrm{MHz}$ frequency range according to Figure 12. The pressure sensor impedance information could be transmitted with little distortion. 


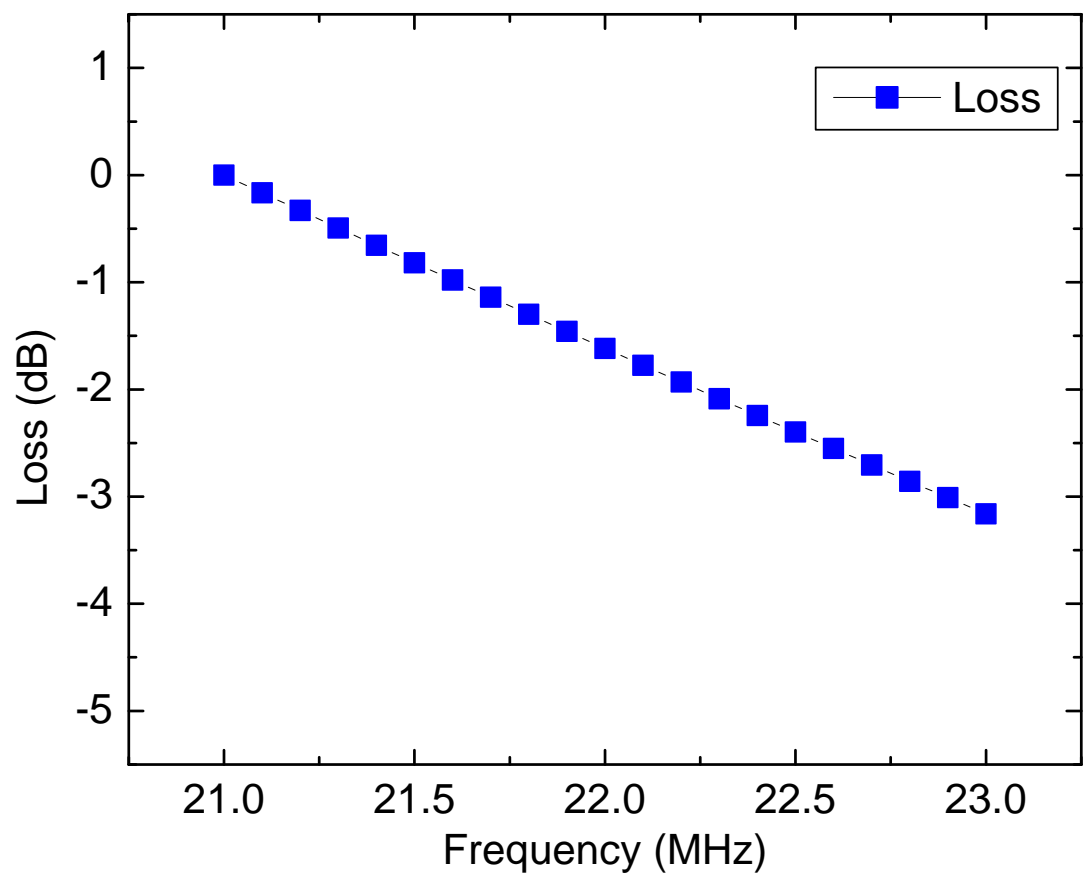

Figure 12: RF signal free-space transmission loss.

\subsection{Long-term stability}

In embedded applications, the sensor stability over a long time is critical. The body implantation makes it very difficult to re-calibrate the sensor readout. To monitor the readout stability, the long-term weight loading experiment is performed. We use the experiment setup in Figure 13. The weight loads are placed on top of the film to apply long-term stable stress.

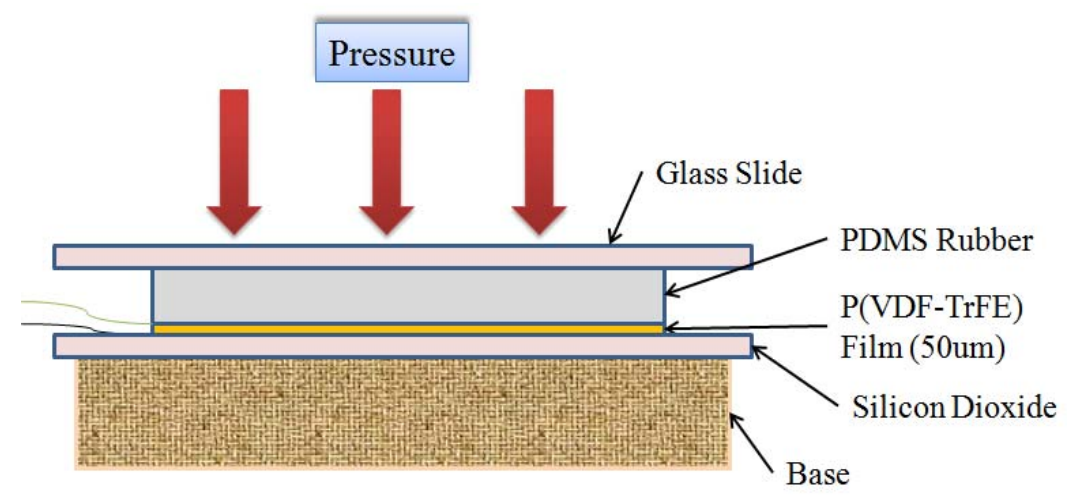

Figure 13: Pressure sensor structure with the P(VDF-TrFE) film. 
A function generator is connected to the resonator film to supply sinusoid stimulus signals near the third harmonic frequency, and an oscilloscope records the averaged RMS voltage across the film in Figure 14. With the help of the modified BVD circuit model, we choose the appropriate series capacitance to enhance the sensitivity, which involves the electromechanical coupling of electrical capacitance and the piezoelectric material. Basically the capacitance should be much smaller than $C_{0}$, usually $1 / 10$, which help boost the resonance. The capacitance value may vary with the film size and the test equipment.

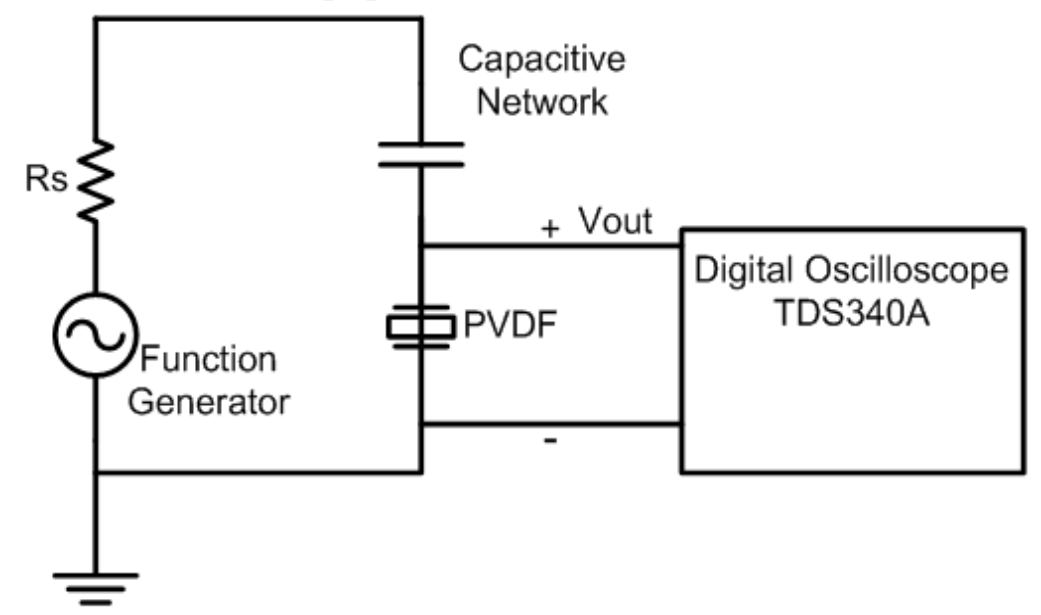

Figure 14: The schematic for measurement. The oscilloscope provides the measurement data.

At room temperature, the sensor long-term drift behavior is monitored and shows good stability. The 13-day long stability measurement in Figure 15 shows negligible drift. 


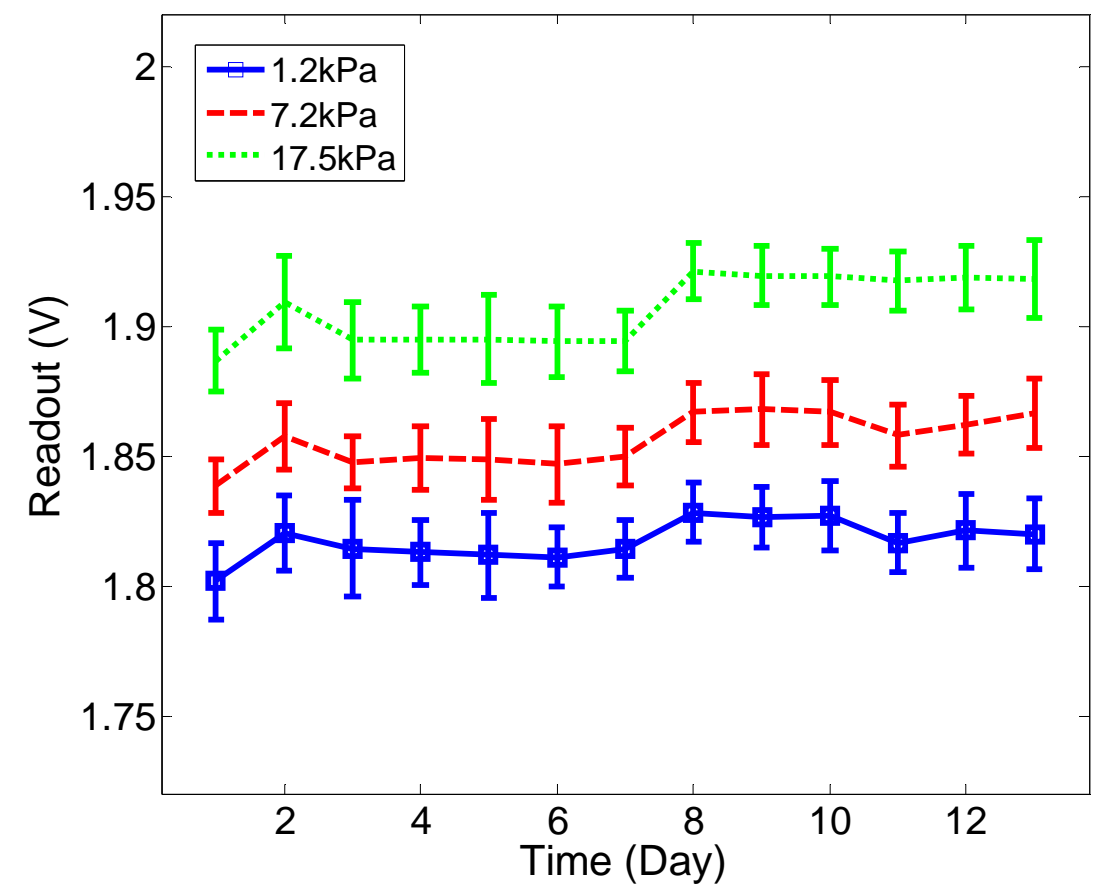

Figure 15: The long-term stability monitored by the RMS voltage reading near resonance.

The small variation possibly comes from the floor vibration. The measurement results can be further improved if better mechanical isolation can be achieved during the experiment. A better sensor packaging design will also help the measurement stability. 


\section{CHAPTER 3}

\section{CONCLUSION AND FUTURE WORK}

In this thesis, we have developed a low-range $\mathrm{P}(\mathrm{VDF}-\mathrm{TrFE})$ copolymer piezoelectric resonance pressure sensor. The BVD equivalent circuit model is used to explain the sensor resonance properties. The calculated results from the model are close to the actual values and can help extract important parameters from a given sensor specification. The proposed sensor exhibits good sensitivity. The lateral-dimension scaling experiment shows the further miniaturization is feasible. The temperature drift characteristic is studied. The $S_{11}$ measurement illustrates a possible solution for integration with a passive RF tag.

The low-range pressure sensor will have a lot of applications in many areas. To be compatible with different sensing environment, special packaging is needed for the sensor. For human implantation, the pressure sensor can be mounted on the tip of the catheter. The $\mathrm{P}(\mathrm{VDF}-\mathrm{TrFE})$ film has the advantage of flexibility, which makes it possible to be fit into any space.

The sensor miniaturization should be further continued following the guide of equivalent circuit model. However, with the scaling down of the film areal size, the difficulty of bonding lead wires to the film surfaces also increases significantly. One of the possible solutions is to add the lead wires when depositing the Au/Pt electrode layer.

Temperature compensation can be done either locally or at the reader side. A dummy pressure sensor identical to the functioning pressure sensor can be placed nearby. The dummy sensor would stand free but experiencing the same temperature with the functioning sensor. Differential measurement will eliminate the temperature drift effect with the help of dummy sensor. People can also design a look-up table for 
the temperature characteristics. The sensor readout data can be calibrated by the lookup table data.

The current problem for the sensor application in liquid environment is the serious damping of resonance peak. The acoustic impedance of $\mathrm{P}(\mathrm{VDF}-\mathrm{TrFE})$ is very close to that of water. Most of the acoustic energy from the piezoelectric resonator radiates into the surrounding medium, which causes the energy loss. To overcome this problem, a buffer layer between $\mathrm{P}(\mathrm{VDF}-\mathrm{TrFE})$ and liquid could be used to increase the energy reflection at the layer interface. Then the majority of acoustic energy is kept within the resonator and the quality factor is improved. 


\section{REFERENCES}

[1] Y. Lee and K. D. Wise, "A batch-fabricated silicon capacitive pressure transducer with low temperature sensitivity," IEEE Trans. Electron Devices, Vol. 29, pp. 42-48 (1982).

[2] M.-X. Zhou, Q.-A. Huang, M. Qin and W. Zhou, "A novel capacitive pressure sensor based on sandwich structures," J. Microelectromechanical Systems, Vol. 14, pp. 1272-1282 (2005).

[3] S. Middelhoek, A. A. Bellekom, U. Dauderstadt, P. J. French, S. R. in 't Hout, W. Kindt, F. Riedijk, and M. J. Vellekoop, "Silicon sensors," Measurement Science and Technology, Vol. 6, pp. 1641-1658 (1995).

[4] L. S. Pakula, H. Yang, H. T. M. Pham, P. J. French and P. M. Sarro, "Fabrication of a CMOS compatible pressure sensor for harsh environment," J. Micromechanics and Microengineering, Vol. 14, pp. 1478-1483 (2004).

[5] A. Mason, N. Yazdi, A. V. Chavan, K. Najafi and K. D. Wise, "A generic multielement microsystem for protable wireless applications," Proceedings of the IEEE, Vol. 86, pp. 1733-1746 (1998).

[6] F. Tseng, C. Yang and L. Pan, "An elastomeric tactile sensor employing dielectric constant variation and applicable to orthodontia," in Proc. IEEE MEMS 2004, pp.564567.

[7] Samaun, K. D. Wise and J. B. Angell, "An IC piezoresistive pressure sensor for biomedical instrumentation," IEEE Trans. Biomedical Engineering, Vol. BME-20, pp. 101-109 (1973).

[8] J. Dargahi, "A piezoelectric tactile sensor with three sensing elements for robotic, endoscopic and prosthetic applications," Sensors and Actuators A, Vol. 80, pp. 23-30 (2000). 
[9] S. Sokhanvar, J. Dargahi and M. Packirisamy, "Influence of friction on piezoelectric sensors," Sensors and Actuators A, Vol. 141, pp. 120-128 (2008).

[10] K. Takashima, S. Horie, T. Mukai, K. Ishida and K. Matsushige, "Piezoelectric properties of vinylidene fluoride oligomer for use in medical tactile sensor applications," Sensors and Actuators A, Vol. 144, pp. 90-96 (2008).

[11] A. V. Shirinov and W. K. Schomburg, "Pressure sensor from a PVDF film," Sensors and Actuators A, Vol. 142, pp. 48-55 (2008).

[12] K. T. Park, R. D. Klafter and P. E. Bloomefield, "A charge readout algorithm for piezoelectric force transducers," in Proc. IEEE ISAF 1986, pp.715-717.

[13] M. Lord and D. M. Smith, "Static response of a simple piezoelectric load cell," J. Biomedical Engineering, Vol. 5, pp.162 (1983).

[14] C. Behling, R. Lucklum and P. Hauptmann, "Response of quartz-crystal resonators to gas and liquid analyte exposure," Sensors and Actuators A, Vol. 68, pp. 388-398 (1998).

[15] L. Ngalamou, N. Noury, E. Chamberod and Ph. Benech, "Analysis of the sensitivity and the temperature influence of a static force sensor based on a PVDF resonator," Sensors and Actuators A, Vol. 57, pp. 173-177 (1996).

[16] F. Kocer and M. P. Flynn, "A new transponder architecture with on-chip ADC for long-range telemetry applications," IEEE J. Solid-State Circuits, Vol. 41, pp. 11421148 (2006).

[17] S. J. Martin, V. E. Granstaff and G. C. Frye, "Characterization of a quartz crystal microbalance with simultaneous mass and liquid loading," Analytical Chemistry, Vol. 63, pp. 2272-2281 (1991).

[18] K. K. Kanazawa and J. G. Gordon, "Frequency of a quartz microbalance in contact with liquid," Analytical Chemistry, Vol. 57, pp. 1770-1771 (1985). 
[19] G. Piazza, P. J. Stephanou and A. P. Pisano, "Piezoelectric aluminum nitride vibrating contour-mode MEMS resonators," J. Microelectromechanical Systems, Vol. 15, pp. 1406-1418 (2006).

[20] C. E. Reed, K. K. Kanazawa and J. H. Kaufman, "Physical description of a viscoelastically loaded AT-cut quartz resonator," J. Appl. Physics, Vol. 68, pp. 19932001 (1990).

[21] S. Ozeri and D. Shmilovitz, "Static Force Measurement by Piezoelectric Sensors," in Proc. IEEE ISCAS 2006, pp. 5179-5182.

[22] D. A. Buttry and M. D. Ward, "Measurement of interfacial processes at electrode surfaces with the electrochemical quartz crystal microbalance," Chemical Reviews, Vol. 92, pp. 1355-1379 (1992).

[23] A. Arnau, T. Sogorb and Y. Jimenez, "Circuit for continuous motional series resonant frequency and motional resistance monitoring of quartz crystal resonators by parallel capacitance compensation," Review of Scientific Instruments, Vol. 73, pp. 2724-2737 (2002).

[24] P. Vanysek and L. A. Delia, "Impedance characterization of a quartz crystal microbalance," Electroanalysis, Vol. 18, pp. 371-377 (2006).

[25] L. F. Brown and D. L. Carlson, "Ultrasound transducer models for piezoelectric polymer films," IEEE Trans. Ultrason. Ferroelec. Freq. Contr., Vol. 36, pp. 313-318 (1989).

[26] R. Lucklum and P. Hauptmann, "The quartz crystal microbalance: mass sensitivity, viscoelasticity and acoustic amplification," Sensors and Actuators B, Vol. 70, pp. 30-36 (2000).

[27] L. Bui, H. J. Shaw and L. T. Zitelli, "Study of acoustic wave resonance in piezoelectric PVF2 film," IEEE Trans. Son. Ultrason., Vol. SU-24, 5, pp. 331-336 (1977). 
[28] H. Chandrahalim, S. A. Bhave, R. G. Polcawich, J. Pulskamp, D. Judy, R. Kaul and M. Dubey, "Influence of silicon on quality factor, motional impedance and tuning range of PZT-transduced resonators," in Technical Dig. Solid State Sensor, Actuator and Microsystems Workshop 2008, pp. 360-363.

[29] I. Jalaly and I. D. Robertson, "Capacitively-tuned split microstrip resonators for RFID barcodes," in Proc. European Microwave Conf. 2005, Vol. 2, pp. 4-7.

[30] Stevan Preradovic, et. al., "Multiresonator-based chipless RFID system for lowcost item tracking," IEEE Trans. on Microwave Theory and Techniques, Vol. 57, pp.1411-1419 (2009).

[31] D. Hermelin, W. Daniaux, S. Ballandras and B. Belgacem, "Fabrication of surface acoustic wave wireless pressure sensor," in Proc. IEEE International Frequency Control Symposium 2009, pp. 96-99.

[32] R. Steindl, A. Pohl, and F. Seifert, "Impedance loaded SAW sensors offer a wide range of measurement opportunities," IEEE Trans. on Microwave Theory and Techniques, Vol. 47, pp. 2625-2629 (1999).

[33] G. Schimetta, F. Dollinger and R. Weigel, "A wireless pressure-measurement system using a SAW hybrid sensor," IEEE Trans. on Microwave Theory and Techniques, Vol. 48, pp. 2730-2735 (2000).

[34] P. V. Nikitin and K. V. S. Rao, "Theory and measurement of backscattering from RFID tags," IEEE Antennas and Propagation Magazine, Vol. 48, pp. 212-218 (2006). [35] N. Lenin and P. H. Rao, "Broadband printed square slot loop antenna," in Proc. IEEE Antennas and Propagation Society International Symposium 2005, Vol. 1B, pp. 557-560. 